\title{
History of syncope predicts loss of consciousness after head trauma: Retrospective study
}

\author{
Dorota Zyśko스, Richard Sutton², Dariusz Timler ${ }^{3}$, Stanisław Furtan ${ }^{4}$, \\ Olle Melander ${ }^{5}$, Artur Fedorowski ${ }^{5,6}$
}

\begin{abstract}
${ }^{1}$ Department of Medical Emergency, Wroclaw Medical University, Wroclaw, Poland
${ }^{2}$ National Heart and Lung Institute, Imperial College, St. Mary's Hospital Campus, London, United Kingdom ${ }^{3}$ Department of Emergency Medicine and Disaster Medicine, Medical University of Lodz, Poland

${ }^{4}$ Department of Emergency Medicine, Zgorzelec County Hospital,

University of Medical Sciences, Legnica, Poland

${ }^{5}$ Department of Clinical Sciences, Lund University, Clinical Research Center,

Skåne University Hospital, Malmö, Sweden

${ }^{6}$ Department of Cardiology, Skåne University Hospital, Malmö, Sweden
\end{abstract}

\begin{abstract}
Background: Head trauma may present as transient loss of consciousness (TLOC) currently classified as traumatic in origin, in contrast to non-traumatic forms, such as syncope. Whether past history of syncope predisposes to loss of consciousness after head injury has been poorly studied.

Methods: A retrospective analysis of data obtained from 818 consecutive patients admitted to Emergency Departments was conducted. Face-to-face semi-structured interviews were performed, where patients' past history of syncope and head injury were explored. Head injury events were stratified as high- or low-energy trauma. Data regarding past syncopal events were explored in regard to number, age at the first occurrence, and syncope circumstances. Multivariate logistic regression model was applied to assess the relationship between loss of consciousness during head injury and past history of syncope.

Results: Both past history of non-traumatic TLOC (odds ratio [OR] 3.78; 95\% confidence interval [CI] 2.13-6.68, $p<0.001)$ and high-energy mechanism (OR 3.84; 95\% CI 2.35-6.28, $p<0.001)$ predicted TLOC after head trauma. This relationship was even stronger when past episodes of TLOC were limited to those typical for reflex syncope (OR 4.34; 95\% CI 2.34-7.89, $p<0.001)$. Further, the number of non-traumatic TLOC episodes in the patient's history was also predictive of TLOC after head injury (OR per 1 episode: 1.24; 95\% CI 1.04-1.48, $p=0.015$ ).

Conclusions: Syncope in a patient's history predicts loss of consciousness after head injury. The clinical importance of this finding merits further investigation. (Cardiol J 2014; 21, 6: 674-678)
\end{abstract}

Key words: head trauma, emergency department, loss of consciousness, syncope, vasovagal syncope

Address for correspondence: Dorota Zyśko, MD, PhD, Department of Medical Emergency, Wroclaw Medical University, ul. Bartla 5, 51-618 Wrocław, Poland, tel/fax: +48 7134593 24, e-mail: dzysko@wp.pl 


\section{Introduction}

Traumatic head injuries tend to cause concussion, which is typically not a transient loss of consciousness (TLOC). However, head trauma may also present as TLOC and currently such loss of consciousness is classified as traumatic in origin, in contrast to non-traumatic forms [1]. The mechanism of this concussive TLOC caused by head trauma is thought to be a 'functional deafferentation' of the cortex as a consequence of diffuse mechanically induced depolarization and synchronized discharge of cortical neurons [2, 3]. It is well known that injuries, both serious and minor, of other parts of the body than the head may provoke TLOC of reflex origin. Blood drawing, injection, piercing, and orthopedic arthroscopy have been reported to induce vasovagal syncope [4-6]. A tilt testing protocol involving subcutaneous insertion of a needle into the dorsum of hand after $10 \mathrm{~min}$ of passive tilt has been shown to have similar to conventional tilt protocols sensitivity and specificity [7]. Further, factors, which often accompany head injury such as sudden and severe emotional distress, pain, sight of blood and wounding, have the potential to provoke reflex syncope [4].

It is estimated that about $20 \%$ of all head injuries are accompanied by TLOC [8]. However, the exact proportion is not known and may vary depending on the selection criteria. As the awareness about dangers related to head trauma is growing, the increasing number of patients with head injury has been recently observed at Emergency Departments (ED) [9]. The occurrence of TLOC as a result of head trauma has been associated with the increased risk of intracranial injury. However, many without intracranial injury sustain TLOC during minimal or mild head trauma, which diminishes the predictive value of TLOC for intracranial damage [10-12]. The aim of this study was to explore the past history of syncope and head injuries among consecutive ED patients. We hypothesized that past history of syncope may predict loss of consciousness after head trauma.

\section{Methods}

A retrospective analysis of data obtained from 818 consecutive patients admitted to ED of 2 acute hospitals in Poland was conducted. The regional hospital in Zgorzelec $(n=603$, September 1 - December 31, 2012) and the Copernicus Memorial Hospital in Lodz $(\mathrm{n}=215$, January 3 - January 24, 2014) contributed sequentially to the data collection. Unconscious patients and those with altered mental state were excluded, and 1 patient refused participation. Face-to-face semi-structured interviews were performed. Patients were asked about their past history of syncope and head injury. The following data were collected: the cause of admission to the ED, the history of past head injuries and their circumstances: age, the total number of past head injuries, the mechanisms of head injuries, signs and symptoms related to head trauma (headache, loss of consciousness, amnesia, nausea/vomiting, bleeding from nose or ear) and how the head injury was managed (medical consultation, suture of wounds, head computerized tomography or magnetic resonance imaging, hospitalization, and surgery). In case the patient reported more than 1 event of head trauma, each event was separately assessed. Each event of head injury was stratified as being a high- (high-speed road traffic accident either as pedestrian, cyclist or vehicle occupant, fall from a height, high-speed injury from a projectile or other object, beating, etc.) or low-energy trauma (falling from upright position i.e. at ground level or low-speed injury from an object) according to the literature [12-14]. Data regarding past syncopal events were explored in regard to number, age at the first occurrence and syncope circumstances (i.e. prolonged standing, rapid standing, instrumentation, injection, blood sight, pain, emotional distress, or no apparent reason). If syncope scenario was that of a typical vasovagal reflex (i.e. involving typical triggers and prodrome), the past episode of TLOC was classified as vasovagal [1].

The Bioethical Commission of the Medical University of Lodz approved the study protocol.

\section{Statistical analysis}

The continuous variables were presented as means and standard deviations or medians and interquartile range depending on their mode of distribution. The categorical variables were presented as numbers and percentages. The past head trauma events related to syncope or epilepsy were excluded from the analysis. Logistic regression model was applied to assess the relationship between loss of consciousness during head injury and past history of syncope. In the multivariate-adjusted model the following confounders were entered: age, gender, and high-energy trauma. P-value $<0.05$ was regarded as significant.

\section{Results}

The clinical characteristics of study group $(\mathrm{n}=818)$ are shown in Table 1 . A total of 319 
Table 1. The clinical characteristics of the study population ( $\mathrm{n}=818$ ).

\begin{tabular}{lc}
\hline Characteristic & $\begin{array}{c}\text { No. (\%) or } \\
\text { mean (SD) }\end{array}$ \\
\hline Male gender & $463(56 \%)$ \\
Age [years] & $41.2 \pm 23.3$ \\
Epilepsy & $13(1.6 \%)$ \\
History of head trauma & $319(39.0 \%)$ \\
History of non-traumatic TLOC & $175(21.4 \%)$ \\
$\begin{array}{l}\text { History of typical vasovagal } \\
\text { syncope }\end{array}$ & $126(15.4 \%)$ \\
\hline
\end{tabular}

SD — standard deviation; TLOC — transient loss of consciousness
(39.0\%) patients reported previous head injury. As some patients reported more than 1 past incident of head injury, the total number of reported events was 418 . Of these, we excluded $15(3.6 \%)$ events, which were caused by syncope-related falls, and $3(0.7 \%)$, which were due to epileptic seizure (Fig. 1). The main injury mechanism, proportions of patients with positive non-traumatic TLOC history, and proportions of males in each etiological group are presented in Table 2, whereas clinical characteristics and management of the reported head injuries are shown in Table 3.

As can be further seen in Table 1 , a total of $175(21.4 \%)$ patients reported at least 1 past non-

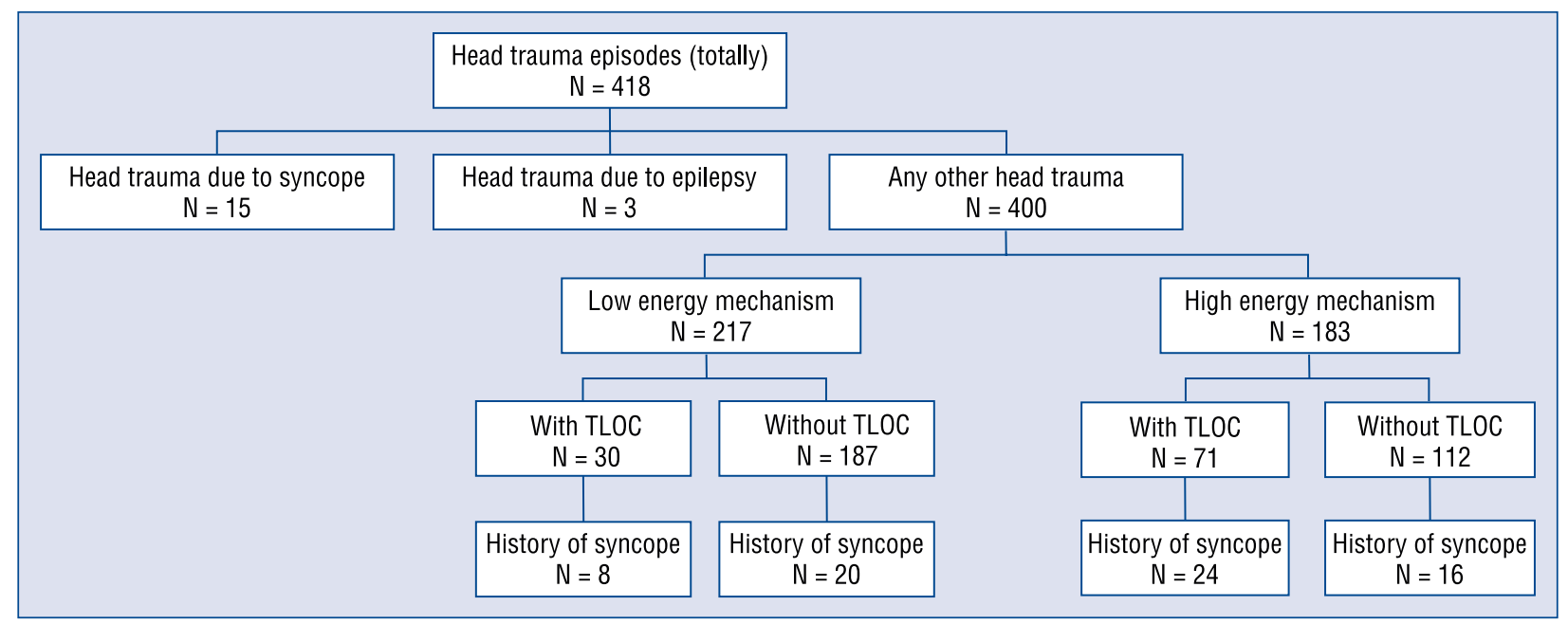

Figure 1. Past episodes of head trauma $(n=418) *$ reported by 319 Emergency Department patients. The episodes were classified according to trauma energy and association (or not) with transient loss of consciousness. The past history of syncope is presented in the last line; *Some patients reported multiple past head trauma episodes, and each episode was assessed separately, hence the higher number of episodes than patients.

Table 2. Past head trauma episodes $(n=400)^{*}$ in relation to the mechanism of head injury and gender reported by 301 of 818 emergency patients.

\begin{tabular}{|c|c|c|c|c|c|c|c|}
\hline \multirow{2}{*}{$\begin{array}{l}\text { Past head } \\
\text { trauma } \\
\text { episodes }\end{array}$} & \multirow[t]{2}{*}{$\begin{array}{c}\text { Total } \\
(n=400)\end{array}$} & \multicolumn{2}{|c|}{$\begin{array}{l}\text { Low-energy mechanism } \\
\qquad(\mathrm{n}=217)\end{array}$} & \multicolumn{4}{|c|}{$\begin{array}{l}\text { High-energy mechanism } \\
\qquad(\mathrm{n}=183)\end{array}$} \\
\hline & & $\begin{array}{l}\text { Fall from } \\
\text { upright } \\
\text { position at } \\
\text { ground level } \\
\text { (n = 99) }\end{array}$ & $\begin{array}{c}\text { Low-speed } \\
\text { injury } \\
(n=118)\end{array}$ & $\begin{array}{l}\text { Road traffic } \\
\text { accident } \\
(n=62)\end{array}$ & $\begin{array}{l}\text { Fall from } \\
\text { a height } \\
\geq 6 \mathrm{~m} \\
(\mathrm{n}=11)\end{array}$ & $\begin{array}{l}\text { Fall from } \\
\text { a height } \\
<6 \mathrm{~m} \\
\text { (n=58) }\end{array}$ & $\begin{array}{c}\text { High-speed } \\
\text { injury, } \\
\text { beating } \\
\text { (n= 52) }\end{array}$ \\
\hline $\begin{array}{l}\text { TLOC after } \\
\text { head trauma }\end{array}$ & $101(25 \%)$ & $19(19.2 \%)$ & $11(9.3 \%)$ & $28(45.9 \%)$ & $7(63.6 \%)$ & $18(31.0 \%)$ & $18(34.0 \%)$ \\
\hline $\begin{array}{l}\text { Past history } \\
\text { of non-trau- } \\
\text { matic TLOC }\end{array}$ & $68(17 \%)$ & $18(18.2 \%)$ & $10(8.5 \%)$ & $17(27.5 \%)$ & $3(27.3 \%)$ & $11(19.0 \%)$ & $9(17.3 \%)$ \\
\hline Male gender & $286(71.5 \%)$ & $60(60.6 \%)$ & $98(83.1 \%)$ & $38(61.3 \%)$ & $10(90.9 \%)$ & $39(67.2 \%)$ & $41(78.9 \%)$ \\
\hline
\end{tabular}

TLOC — transient loss of consciousness; *Some patients reported multiple past head trauma episodes, and each episode was assessed separately, hence the higher number of episodes than patients; head-trauma episodes caused by epilepsy or primary syncope were excluded ( $n=18$ ) 
Table 3. Clinical characteristics of past episodes of head injury $(n=400)^{*}$ reported by 301 of 818 emergency patients presented as numbers and percentages.

\begin{tabular}{lc}
\hline Characteristic & No. (proportion in \%) \\
\hline Loss of consciousness & $101(25.3 \%)$ \\
Retrograde amnesia & $94(23.5)$ \\
Vomiting & $44(11.0 \%)$ \\
Orbital hematoma & $15(3.8 \%)$ \\
Otorrhagia & $1(1.3 \%)$ \\
Epistaxis & $25(6.3 \%)$ \\
Wounds requiring sutures & $166(41.5 \%)$ \\
Medical attention sought & $320(80.0 \%)$ \\
Head CT/ MRI & $68(17.0 \%)$ \\
X-ray & $132(33.0 \%)$ \\
Hospitalization & $102(25.5 \%)$ \\
Hospitalization in ICU & $15(3.8 \%)$ \\
Neurosurgery & $3(0.8 \%)$ \\
\hline
\end{tabular}

$\mathrm{CT}$ - computerized tomography; MRI - magnetic resonance imaging; ICU — intensive care unit; *Some patients reported multiple past head-trauma episodes, and each episode was assessed separately, hence the higher number of episodes than patients; head-trauma episodes caused by epilepsy or primary syncope were excluded $(n=18)$

-traumatic episode of TLOC. The median number of past TLOC episodes was 2 (interquartile range [IQR] 1-4), and the median age of the first reported episode was 26.5 years (IQR 15-54).

\section{Multivariate analysis}

Both past history of non-traumatic TLOC (odds ratio [OR] 3.78; 95\% confidence interval [CI] 2.13-6.68, $\mathrm{p}<0.001$ ) and high-energy mechanism of head injury (OR 3.84, 95\% CI 2.35-6.28, p < $<0.001$ ) predicted loss of consciousness after head trauma. The relationship between positive syncope history and loss of consciousness due to head trauma was even stronger when past syncope episodes were limited to those with typical vasovagal reflex pattern (OR 4.34, 95\% CI 2.34-7.89, p < 0.001). Further, the number of non-traumatic TLOC episodes in the patient's history was also predictive of TLOC after head injury (OR per 1 episode 1.24, $95 \%$ CI 1.04-1.48, $\mathrm{p}=0.015$ ).

\section{Discussion}

The main finding of the study was that history of vasovagal (reflex) syncope predicts loss of consciousness after head injury independently of age, gender, and the mechanism of injury. The exact mechanism of a particular loss of consciousness after head trauma cannot be established in a retrospective study. However, if the estimated trauma energy is comparably low, the higher incidence of loss of consciousness in patients with past history of syncope compared with those without suggests other than purely traumatic mechanism. Pain, emotional stress, and sight of blood, all present during the head injury, are typical vasovagal reflex triggers [1]. We, therefore, propose that higher fainting rate after head trauma among patients with positive syncope history depends on the increased reflex susceptibility indicated by past syncope events, in contrast to high-energy trauma that leads to unconsciousness, which may be relatively transient, by concussion and not via a reflex mechanism. Injuries are the third most common cause of death after cardiovascular disease and cancer. About half of the deaths due to injuries occur as a result of head injuries [12]. The number of hospital admissions due to head injury is approximately $250-300 / 100,000$ per year, and the number of patients with head injury not meriting admission is many times greater [9, 12, 15]. Loss of consciousness, amnesia, headache, vomiting, earlier abuse of alcohol are risk factors for intracranial injury and constitute indications for computerized tomography of head. In this context, loss of consciousness after head trauma, although considered to be a very important symptom, has a surprisingly low predictive value for determining clinically significant brain injury [10, 12, 16-18] In this study, $25 \%$ of head trauma episodes led to unconsciousness. The higher incidence of TLOC after head trauma ( $>30 \%$ ) observed by Roitman [19] may be explained by a higher proportion of traffic accidents (high-energy) in that cohort compared with our study. In another study, where head trauma was due to traffic accidents in $10 \%$ cases only, loss of consciousness was observed in fewer patients (20\%) than in our study [20].

Syncope after head trauma seems to be more frequent than that during medical procedures. However, among various medical procedures, the rate of syncopal events depends on the type of procedure being about $0.01 \%$ for vaccination, and $1 \%$ for colonoscopy or transthoracic pleural biopsy, up to $5.1 \%$ during diagnostic injection [21-24]. It may be presumed that head trauma evokes a high level of emotion, especially if associated with skin injury, pain, bleeding etc. being more prone to provoke reflex syncope than vaccination or professional sampling of blood. Interestingly, a belief of significant blood loss is associated with increased risk of vasovagal symptoms during blood donation, irrespective of actual blood loss [25]. 
This study throws light on the difficult ED question of whether the loss of consciousness during head injury may be applied as a marker of trauma severity. In many cases this is an easy question to answer but in some the question is crucial to the proper management of patient. If there is a previous history of reflex syncope in unclear trauma circumstances, consideration must be given that syncope has recurred due to head trauma, which operated as a reflex trigger, and, accordingly, a different management approach will be needed. However, the trauma scenario may be more complicated. An example of such an event might be a patient found unconscious at the bottom of a flight of stairs. A head injury has been sustained and the patient is unable to explain the sequence of events leading to the trauma. Even if there is a previous history of reflex syncope, testing may be indicated to ascertain the most probable mechanism of trauma and/or syncope, perhaps by implantation of an electrocardiographic loop recorder.

\section{Limitations of the study}

The main limitation of the study is its retrospective character and its inherent characteristics: missing or incomplete data resulting from difficulty in recalling facts. Two particular limitations are thereby implied. The first is that as there is no 'gold standard' for syncope diagnosis from the history, there is a limitation in precision of diagnosis and, secondly, in the cases of reflex syncope diagnosis this same limitation is also pertinent. The severity and site of traumatic brain injury were not assessed because only retrospective data regarding the circumstances of head injuries and their clinical presentations were collected. For the same reason, the information describing history of drug or/and alcohol dependence as well Glasgow Coma Scale were not collected. Studying patients at the ED has probably limited the study sample to those who are more active in observing and giving attention to their medical problems.

\section{Conclusions}

1. Syncope in a patient's history is an independent predictor of loss of consciousness after a head injury.

2. The clinical importance of this finding merits further investigation.

Conflict of interest: None declared

\section{References}

1. Moya A, Sutton R, Ammirati F et al. Task Force for the Diagnosis and Management of Syncope; European Society of Cardiology (ESC); European Heart Rhythm Association (EHRA); Heart Failure Association (HFA); Heart Rhythm Society (HRS). Guidelines for the diagnosis and management of syncope (version 2009). Eur Heart J, 2009; 30: 2631-2671.

2. Kushner D. Mild traumatic brain injury: Toward understanding manifestations and treatment. Arch Intern Med, 1998; 158: 1617-1624.

3. Shaw NA. The neurophysiology of concussion. Prog Neurobiol, 2002; 67: 281-344

4. Kenny RA, Bhangu J, King-Kallimanis BL. Epidemiology of syncope/ /collapse in younger and older Western patient populations. Prog Cardiovasc Dis, 2013; 55: 357-363.

5. Vieira EP, Ribeiro AL, Pinheiro Jde J, Alves Sde M Jr. Oral piercings: immediate and late complications. J Oral Maxillofac Surg, 1022; 69: 3032-3037.

6. Song SY, Roh WS. Hypotensive bradycardic events during shoulder arthroscopic surgery under interscalene brachial plexus blocks. Korean J Anesthesiol, 2012; 62: 209-219.

7. Adamec I, Mišmaš A, Zaper D, Junaković A, Hajnšek S, Habek M. Short pain-provoked head-up tilt test for the confirmation of vasovagal syncope. Neurol Sci, 2013; 34: 869-873.

8. Barrow A, Ndikum J, Harris T. Late presentations of minor head injury. Emerg Med J, 2012; 29: 983-988.

9. Marin JR, Weaver MD, Yealy DM, Mannix RC. Trends in visits for traumatic brain injury to emergency departments in the United States. JAMA, 2014; 311: 1917-1919.

10. Pandor A, Harnan S, Goodacre S, Pickering A, Fitzgerald P, Rees A. Diagnostic accuracy of clinical characteristics for identifying CT abnormality after minor brain injury: a systematic review and meta-analysis. J Neurotrauma, 2012; 29: 707-718.

11. Abdul Rahman YS, Al Den AS, Maull KI. Prospective study of validity of neurologic signs in predicting positive cranial computed tomography following minor head trauma. Prehosp Disaster Med, 2010; 25: 59-62.

12. National Institute for Clinical Excellence. NICE web site. http:// //www.nice.org.uk/nicemedia/live/14355/66328/66328.pdf (accessed 1/6/2014).

13. Kara H, Bayir A, Ak A et al. Trauma in elderly patients evaluated in a hospital emergency department in Konya, Turkey: A retrospective study. Clin Interv Aging, 2014; 9: 17-21.

14. Içer M, Güloğlu C, Orak M, Ustündağ M. Factors affecting mortality caused by falls from height. Ulus Travma Acil Cerrahi Derg, 2013; 19: 529-535.

15. Vos PE, Alekseenko Y, Battistin L et al. European Federation of Neurological Societies. Mild traumatic brain injury. Eur J Neurol, 2012; 19 191-198.

16. Sharif-Alhoseini M, Khodadadi H, Chardoli M, Rahimi-Movaghar V. Indications for brain computed tomography scan after minor head injury. J Emerg Trauma Shock, 2011; 4: 472-476.

17. Sheehan A, Batchelor JS. A retrospective cohort study to re-evaluate clinical correlates for intracranial injury in minor head injury. Emerg Med J, 2012; 29: 899-901.

18. Wolf H, Machold W, Frantal S et al. Risk factors indicating the need for cranial CT scans in elderly patients with head trauma: An Austrian trial and comparison with the Canadian CT Head Rule. J Neurosurg, 2014 120: 447-452.

19. Roitman P, Gilad M, Ankri YL, Shalev AY. Head injury and loss of consciousness raise the likelihood of developing and maintaining PTSD symptoms. J Trauma Stress, 2013; 26: 727-734.

20. Leong LB, Sukarom S, Vasu A, Hian LG. Identifying predictors of an abnormal computed tomographic scan among patients with a head injury and a Glasgow Coma Scale of 15. Eur J Emerg Med, 2013; 20: 86-90.

21. Son HS, Lee SH, Darlong LM et al. Is there a role for a needle thoracoscopic pleural biopsy under local anesthesia for pleural effusions? Korean J Thorac Cardiovasc Surg, 2014; 47: 124-128.

22. Thomas-Gibson S, Thapar C, Shah SG, Saunders BP. Colonoscopy at a combined district general hospital and specialist endoscopy unit: Lessons from 505 consecutive examinations. J R Soc Med, 2002; 95: 194-197.

23. Zervou EK, Ziciadis K, Karabini F, Xanthi E, Chrisostomou E, Tzolou A. Vasovagal reactions in blood donors during or immediately after blood donation. Transfus Med, 2005; 15: 389-394

24. Kennedy DJ, Schneider B, Casey E et al. Vasovagal rates in flouroscopically guided interventional procedures: a study of over 8,000 injections. Pain Med, 2013; 14: 1854-1859.

25. Ditto B, Balegh S, Gilchrist PT, Holly CD. Relation between perceived blood loss and vasovagal symptoms in blood donors. Clin Auton Res, 2012; 22: 113-116. 